\title{
Fomento de iniciativas de emprendimiento y la innovación para acercar a los jóvenes universitarios al mundo empresarial
}

Promotion of entrepreneurship and innovation initiatives to bring young university students closer to the business world

Edison Becerra Molina ${ }^{1}$

Recibido: 15-07-2020 / Revisado: 13-08-2020 / Aceptado: 10-09-2020 / Publicado: 03-10-2020

\begin{abstract}
.
DOI: $\underline{\text { https://doi.org/10.33262/cienciadigital.v4i4.1430 }}$

The study is oriented towards the description of the administrative and academic processes in terms of the ideas, purposes and problems that revolve around entrepreneurship, for which it was necessary to develop projects, programs and an integrated plan for UCACUE, which allows to contribute to the development of the entrepreneurial culture in the training of professional leaders and entrepreneurs as one of the transversal axes of the University's processes, through the design of the institutional strategy for research, development, innovation and incubation and contribute to the construction of learning processes, based on the creation of added value in the curricular and extracurricular academic processes, considering the University policies linked to the sustainable development of the territory. Among the conclusions of the information collected with respect to the semi-structured interviews with the main authorities of the Catholic University of Cuenca, it allowed to determine that the dimension of the academic management model, in the Academic Units of the Catholic University of Cuenca, the three authorities coincide that there are policies, and that the University must promote entrepreneurship and innovation.

The purpose was to collect information from the main authorities of UCACUE, to carry out a doctoral research work in education, the interview was directed to the Authorities of the Catholic University of Cuenca, to improve institutional processes, which aims to build a theoretical-academic management model for the development of innovative entrepreneurship policies and programs at the Universidad Católica de
\end{abstract}

${ }^{1}$ Universidad Católica de Cuenca, Cuenca, Ecuador, jbecerram@ucacue.edu.ec 
Cuenca, which contributes to the professional profile in the different careers in correspondence with the economic-social demand of the territory of zone 6 , in each one of the races.

Keywords: University, Curriculum, entrepreneurship, innovation and training.

\section{Resumen.}

El estudio se orienta en la descripción de los procesos administrativos y académicos en cuanto a las ideas, propósitos y problematizaciones que giran alrededor del emprendimiento, para lo cual fue necesario desarrollar los proyectos, programas y un plan integrado para la UCACUE, que permita contribuir al desarrollo de la cultura emprendedora en la formación de profesionales líderes y emprendedores como uno de los ejes transversales de los procesos de la Universidad, mediante el diseño de la estrategia institucional de investigación, desarrollo innovación e incubación y aportar a la construcción de procesos de aprendizaje, basado en la creación de valor agregado en los procesos académicos curriculares y extracurriculares, considerando las políticas Universitarias vinculadas al desarrollo sostenible del territorio. Entre las conclusiones de la información recogida con respecto a las entrevistas semiestructuradas a las principales autoridades de la Universidad Católica de Cuenca, permitió determinar que la dimensión del Modelo de gestión académica, en las Unidades Académicas de la Universidad Católica de Cuenca, las autoridades coinciden que existen políticas, y que la Universidad debe impulsar el emprendimiento y la innovación.

El propósito fue recabar información de las principales autoridades de la UCACUE, para la realización de un trabajo de investigación doctoral en educación, la entrevista fue dirigido a las Autoridades de la Universidad Católica de Cuenca, para mejorar los procesos institucionales, que tiene por objeto construir un modelo de gestión teórico- académico para el desarrollo de políticas y programas de emprendimiento innovador en la Universidad Católica de Cuenca, que aporte al perfil profesional en las diferentes carreras en correspondencia con la demanda económico- social del territorio de la zona 6 , en cada una de las carreras.

Palabras claves: Universidad, Currículo, emprendimiento, innovación y formación.

\section{Introducción.}

Las políticas de Emprendimiento e Innovación nacen con el objetivo de instituir sinergias mediante el trabajo articulado, armónico y coordinado de los actores del ecosistema de emprendimiento e innovación. Por ello con un trabajo conjunto y alineado entre la academia, los sectores productivos y el gobierno, permitirá convertir al Ecuador en uno de los países más atrayentes para emprender, por tanto, generará nuevos emprendimientos con gran potencial de exportación, nuevas fuentes de trabajo decente, mejores oportunidades para los 
ciudadanos; y, desarrollo y adelanto sostenible para el país, en esta época difícil de emergencia sanitaria por la presencia del COVID, como estrategia para la reactivación económica de la región y el país.

En consecuencia, se hace necesario tener un horizonte de desarrollo humano integral y sustentable, en donde la academia considere a la educación integral, así como la generación de conocimiento que aporte a la transformación social y productiva del país, además que ejerza de manera responsable los procesos de formación en las competencias y el rol que deben ejercer los estudiantes y egresados de las Unidades Académicas para contribuir en la construcción de una sociedad más justa, de allí que el emprendimiento es un eje de formación que pueda articular acciones y contribuciones a la generación de propuestas innovadoras de impacto social que propendan a resolver las problemáticas que el país enfrenta.

El presente trabajo de investigación se encuentra centrado en el eje transversal de la cultura emprendedora, la política de emprendimiento innovador, los elementos curriculares y las competencias, necesarias para generar un modelo de gestión teórico-académico para el desarrollo de políticas y programas de emprendimiento innovador en la Universidad Católica de Cuenca-UCACUE, que aporte a la formación de nuevos emprendedores en las diferentes carreras en correspondencia con la demanda económico- social del territorio de la zona 6.

El estudio partió de la descripción de los procesos administrativos y académicos en cuanto a las ideas, propósitos y problematizaciones que giran alrededor del currículo, bajo el cual se desarrollan actividades, proyectos y programas curriculares y extracurriculares para la UCACUE, que permitieron constituir, establecer, comprobar y programar los objetivos, contenidos, curriculares y extracurriculares a ser implementados en el proceso enseñanzaaprendizaje, que estén articulados con la cultura emprendedora que se sintetizó en la autoría del modelo que se propone a la UCACUE.

\section{Metodología.}

Para el análisis de la información de los participantes del trabajo de investigación, para el caso de las de las entrevistas, la técnica es la entrevista y el instrumento el guión de preguntas semiestructuradas, igual mente se consideró tres fases.

Una primera fase con la variable: Cultura emprendedora, que indica la situación actual con respecto a la dimensión modelo de gestión académica.

La segunda fase proporcionó la variable política de emprendimiento innovador en el modelo de gestión académica, con la dimensión políticas de emprendimiento innovador, estrategias que deben ser contemplados en el modelo para el desarrollo de la cultura emprendedora.

La tercera fase proporciona la variable currículo, con la dimensión elementos curriculares, ligado a las competencias que deben considerarse en el modelo de gestión teórico- académico 
para el desarrollo de políticas y programas de emprendimiento innovador en la Universidad Católica de Cuenca.

Arias (2012) expresa que "Se entenderá por técnica de investigación, el procedimiento o forma particular de obtener datos o información" (p. 53). La técnica utilizada fue la entrevista. Para Tejada (1997), la recolección de datos de es una "Las fases más transcendentales en el proceso de investigación científica” (p. 95). Lo que ha de suponer entonces, uno de los ejes principales de la investigación.

Así mismo, Hurtado (2010), sostiene que la técnica de la entrevista obtiene información a través de preguntas a otras personas, pero:

Se diferencia, porque en la encuesta no se establece un diálogo con el entrevistado y el grado de interacción es menor. La técnica de la encuesta corresponde a un ejercicio de búsqueda de información acerca del evento de estudio, mediante preguntas directas a varias unidades o fuentes (p. 873).

Por su parte, Hernández, Fernández, Baptista (2016) sostienen que las entrevistas:

Implican que una persona calificada (entrevistador) aplica el cuestionario a los participantes; el primero hace las preguntas a cada entrevistado y anota las respuestas. Su papel es crucial, resulta una especie de filtro. El primer contexto que se revisará de una entrevista es el personal ("cara a cara"). Normalmente se tienen varios entrevistadores, quienes deberán estar capacitados en el arte de entrevistar y conocer a fondo el cuestionario. No deberán sesgar o influir en las respuestas (p. 233).

Del mismo modo, Hurtado (2010), afirma que "es un instrumento de investigación de campo que se emplea frente al entrevistado con una serie de preguntas por escrito, concernientes al evento de estudio" (p. 867).

Ante lo expuesto, las entrevistas semiestructuradas son una estrategia mixta pues a pesar de tener como instrumento el guion de entrevista, su diseño flexible permite las preguntas espontáneas producto del diálogo establecido en función del interés de la investigación, pudiendo profundizarse en aspectos significativos de la investigación.

Por su parte, Arias (2012) afirma que el guion de entrevista consiste:

En la modalidad de encuesta que se realiza de forma escrita mediante un instrumento o formato de papel contentivo de una serie de preguntas. Se le denomina cuestionario auto administrado porque debe ser llenado por el encuestado, sin intervención del encuestador (p. 74).

De acuerdo a estas conceptualizaciones, el procedimiento que se siguió para concretar la información a obtener fue la técnica de la entrevista semiestructurada, la misma que estuvo 
compuesto por preguntas relevantes, focalizadas en el objetivo de la presente investigación como es "Construir un modelo de gestión teórico- académico para el desarrollo de políticas y programas de emprendimiento innovador en la Universidad Católica de Cuenca, que aporte al perfil profesional en las diferentes carreras en correspondencia con la demanda económicosocial del territorio de la zona 6, periodo 2014-2018".

Los guiones de entrevistas fueron dirigidas a las Autoridades principales de la Universidad Católica de Cuenca; Rector, Vicerrectora Académica; y, al Señor Vicerrector de Investigación e Innovación. Los enunciados que fueron considerados correspondieron a tópicos inherentes al emprendimiento e innovación, así como la dimensión del Modelo de Gestión Académica con dos preguntas, la dimensión referente a políticas de emprendimiento innovador con tres preguntas y la dimensión elementos curriculares con seis preguntas, de manera tal que los entrevistados pudieran expandirse y expresar sus opiniones y valoraciones sin ningún tipo de interferencia.

El nivel de investigación fue la investigación descriptiva, según, Arias (2016), señala que: "La investigación descriptiva consiste en la caracterización de un hecho, fenómeno, individuo o grupo, con el fin de establecer su estructura o comportamiento" (p.24).

Por su parte, Palella y Martins (2012), al referirse a la validez, lo definen como "La medida en que los resultados de la prueba se asocian con alguna otra medida de la misma aptitud" (p. 277).

De igual manera, Arias (2012) afirma: "La validez del cuestionario significa que las preguntas o ítems deben tener una correspondencia directa con los objetivos de la investigación, es decir las interrogantes consultarán sólo aquello que se pretende conocer o medir" (p. 201).

En tal sentido, se elaboró el instrumento del guion de entrevista, considerando la rúbrica de evaluación y validación de los instrumentos es la que utiliza, la Universidad Católica de Cuenca en la Dirección Académica. Estructurada la rúbrica en categorías (Claridad, coherencia y relevancia), además del puntaje, calificación e indicador.

Para el procesamiento y análisis de la información, se consideró tres fases.

Una primera fase con la variable: Cultura emprendedora, que indica la situación actual con respecto a la dimensión modelo de gestión académica.

La segunda fase proporcionó la variable política de emprendimiento innovador en el modelo de gestión académica, con la dimensión políticas de emprendimiento innovador, estrategias que deben ser contemplados en el modelo para el desarrollo de la cultura emprendedora.

La tercera fase proporciona la variable currículo, con la dimensión elementos curriculares, ligado a las competencias que deben considerarse en el modelo de gestión teórico- académico 
para el desarrollo de políticas y programas de emprendimiento innovador en la Universidad Católica de Cuenca.

El diseño y validación del Modelo de Gestión Teórico- Académico para el Desarrollo de Políticas y Programas de Emprendimiento Innovador en la Universidad Católica de Cuenca, desarrollados por el autor de la presente investigación, fue validado, considerando desde el punto de vista didáctico, los indicadores que tomaron en cuenta los validadores expertos, representados por la Academia, sector Gubernamental y los Sectores Productivos. Los indicadores considerados en la validación fueron la factibilidad curricular, precisión, utilidad, ética, pertinencia y contenidos.

De igual forma, la herramienta estadística para el desarrollo de la investigación, utilizada fue la estadística descriptiva. El análisis de los datos es una parte fundamental de toda investigación, en tal sentido, Tal como señala, Pérez (2016), se trata de una fase de búsqueda sistemática reflexiva de la información que hemos recabado y se ha de llevar a cabo el análisis pretendido sobre el estudio con el fin de expresarlos numérica y gráficamente.

En relación a los procedimientos que condujeron a reunir datos con el propósito específico de estructurar el modelo, se procedió a revisar los datos o respuestas para luego ser analizados. Las contestaciones a los guiones de entrevista se compilaron de forma automática y ordenada en formularios, con figuras y datos de las respuestas en tiempo real, que fueron procesados, analizados e interpretados, considerando el uso de la triangulación, validez, confiabilidad y contrastación de los instrumentos.

\section{Fundamentación Teórica.}

Se considera oportuno mencionar una serie de investigaciones que han servido de sustento para el presente trabajo de investigación, Bajo esta misma temática, Arroyo (2016), realizó una tesis doctoral titulada: Emprendimiento y Universidad emprendedora: conceptualización, propuesta metodológica y caracterización de la Universidad Politécnica de Valencia, presentada en la Universidad Politécnica de Valencia - España, el estudio se centró en la caracterización de las universidades emprendedoras como agentes clave para el desarrollo socioeconómico de sus territorios, partiendo del debate sobre la conceptualización del término emprendimiento y sus implicaciones para la aplicación y análisis empírico.

El objetivo general del estudio estuvo orientado a ofrecer una propuesta metodológica para el análisis y la caracterización de universidades emprendedoras. Para alcanzar este objetivo principal se fijaron una serie de objetivos específicos, como fueron: 1.- La divergencia entre la conceptualización de emprendimiento y su aplicación práctica; 2.- La transformación y consideración de las Universidades emprendedoras y su reestructuración para atender a las nuevas necesidades; 3.- La caracterización de la Universidad Politécnica de Valencia como emprendedora. 
De igual forma, Villar (2015), presentó su tesis doctoral titulada: Los estilos de aprendizaje y su aplicación en la formación para el emprendimiento, presentada en la Universidad de Burgos, España. La tesis tuvo como propósito incidir en el apoyo al emprendimiento, en varios de los puntos claves de la recuperación económica y social: la creación de empresas, el autoempleo, la fijación de expectativas, el aprovechamiento de recursos ociosos, el flujo monetario, la inversión y el consumo.

El paradigma epistémico de la investigación en referencia, se apuntaló en el método cuantitativo, empírico-analítico, el tipo de indagación al que se recurrió en función de los objetivos propuestos, está íntimamente ligado con el diseño de campo y nivel descriptivo, por cuanto el investigador analizó las técnicas de emprendimiento innovador en la Universidad Católica de Cuenca, y su relación con los sectores productivos y las políticas gubernamentales.

Así mismo, la tesis doctoral de Salinas (2008), presentada en la Universidad Tecnológica de Monterrey, México, titulada: Desarrollo de habilidades de innovación y creatividad en estudiantes universitarios de ingenierías. El objetivo general de esta Investigación consistió en indagar cómo se desarrollan competencias de innovación y creatividad en estudiantes Universitarios de ingeniería. Así mismo, fue importante analizar el factor cultural como parte fundamental para el desarrollo de habilidades de innovación y creatividad, así como la comparación del perfil de los estudiantes y los profesores por su nivel de estudios y entre Universidades.

A continuación, se detalla algunas cronológicas de conceptualización de emprendimiento, considerando autores principales.

Según, Schumpeter (2009), sostiene ubica al emprendedor como centro del sistema económico, mencionando que la ganancia viene del cambio, y este es producido por el empresario innovador.

Así mismo, Druker (1985), señala que el emprendimiento es una práctica, es un comportamiento, es decir una conducta y una actitud, por eso, y retomando la idea de lo anteriormente mencionado, se expresa por medio de la educación y se modifican comportamientos, puede entonces señalarse, que existe una estrecha relación entre espíritu emprendedor y educación.

Igualmente, Harbison, (1956), señala que el emprendimiento consiste en las capacidades y habilidades de los emprendedores (sean o no dueños de la empresa).

De igual forma, Carland et al, (1988), sustenta que el emprendimiento es la estrategia empresarial basada en el crecimiento y la innovación. 
Por su parte, Wennekers y Thurik (1999), definen el término emprendedor como aquellos individuos con la capacidad manifiesta y deseo, ya sea por ellos mismos o mediante equipos, dentro o fuera de organizaciones existentes, de crear nuevas oportunidades económicas e introducir sus ideas en los mercados, haciendo frente a la incertidumbre y a otros obstáculos, adoptando decisiones sobre la localización, y la forma y uso de los recursos.

Del mismo modo, Rasheed (2000), considera que la educación en emprendimiento puede afectar los atributos que tengan los individuos y puede forjar actitudes emprendedoras en ellos. Puede promover cualidades psicológicas favorables para la actividad emprendedora, tales como la auto confianza, la autoestima, la auto eficacia y la necesidad de logro. Inclusive, la educación en emprendimiento para los jóvenes, puede colaborar en evitar la generación de actitudes socialmente no deseables, como la vagancia o la delincuencia.

En efecto, el Foro Mundial UNESCO. (2015), considera que el emprendimiento y la innovación ayuden a construir un mundo distinto hasta el 2050.

En lo que respecta a las Naciones Unidas (2012), razona que la Iniciativa empresarial para el desarrollo", son de carácter polifacético y guardan relación con otras esferas como la educación y la capacitación, la tecnología y la innovación, la financiación y el fomento de la capacidad emprendedora.

Por otro lado, la Agenda 2030 y los Objetivos de Desarrollo Sostenible de las Naciones Unidas, (2016), considera que se debería "Promover políticas orientadas al desarrollo que apoyen las actividades productivas, la creación de puestos de trabajo decentes, el emprendimiento, la creatividad y la innovación, y fomentar la formalización y el crecimiento de las microempresas y las pequeñas y medianas empresas".

Por último, Shane y Venkataraman, (2017), El emprendedor es quien descubre, evalúa y explota oportunidades rentables, tomando en cuenta el riesgo, alerta a las oportunidades y necesidad por la innovación.

Por su parte, Pérez (2016), recoge en el plano teórico un conjunto de actividades que las Universidades emprendedoras, llevan a cabo con diferentes agentes sociales con los que se relacionan, orientadas a las necesidades del bienestar social y a cooperar con los objetivos públicos y privados, los cuales llama el autor ejes vertebradores (tercera misión) que son el emprendimiento, la creatividad y la innovación. Indicadores que el investigador considera relevantes para que las Universidades puedan enfrentar los retos y desafíos formativos relacionados con las actividades educativas, presente en el currículo y su relación con la dinámica económico social, desde una visión humanista que se complemente con la actividad comercial, empresarial y productiva para la generación de empleo y bienestar, así como transferir conocimiento tecno científico a la sociedad. 
En cuanto a los ejes de la "tercera misión" implican, según Pérez (2016), una nueva forma de llevar a cabo el proceso de innovación y emprendimiento (I+D), de naturaleza cooperativa o en colaboración con los otros agentes del sistema, así como el diseño de nuevos espacios de transferencia y creación de conocimiento, orientados a la innovación en cualquiera de sus categorías o dimensiones.

Figura 1. La "Tercera Misión” de la Universidad

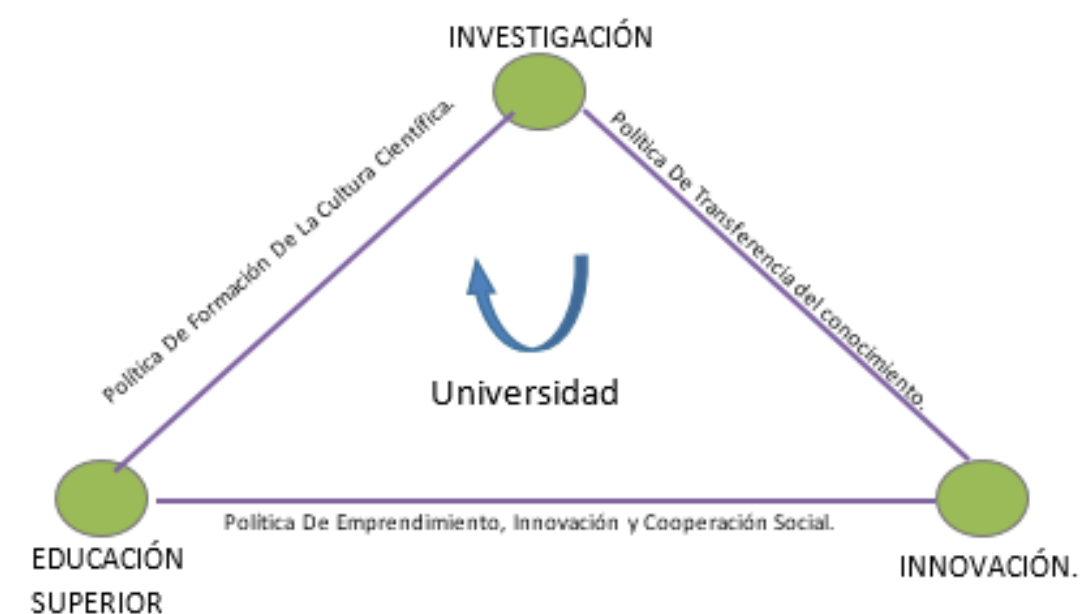

Fuente: Elaboración propia (2020), Basado en los razonamientos de Pérez (2016)

La información recogida permite determinar que, para llevar a cabo esta nueva misión, es necesario dirigir el proceso hacia la generación de cambio, creación de la cultura científica e innovación, así como formar a los asesores y gestores emprendedores como agentes inductores de innovación, todos estos elementos planteados por el actor tienen relevancia teórica y práctica para el desarrollo del modelo de emprendimiento e innovación teórico académico, que fue desarrollado por el investigador para ser presentado a la Universidad Católica de Cuenca.

El diseño de las acciones prenombradas se ha convertido en la nueva habilidad para fortalecer la técnica de innovación de las Universidades emprendedoras, compuesta por labores, en primera instancia ajustada en cómo mejorar y estimular el régimen de educación, para suministrar el desarrollo efectivo de la "tercera misión" de la Universidad; y, la cuarta destinada al impulso de sistemas de transferencia del conocimiento entre Universidades, organismos gubernamentales o públicos de investigación (OPis) e Industrias.

Por otra parte, Salinas (2008), sostiene que se debe tomar en cuenta el ambiente propicio para que los estudiantes desarrollen habilidades de creatividad como la amplitud de respuestas correctas, defensa de ideas, desarrollo de iniciativas del estudiante e independencia de los mismos, con el objeto de formar estudiantes críticos, participativos, reflexivos, tecnológicos e innovadores, en el marco de una cultura de paz y de desarrollo sostenible, donde la eco pedagogía juega un papel importante, pero también la dimensión emprendedora, para que los 
futuros profesionales, asuman con creatividad, con el apoyo del Estado el riesgo de crear empresas generadoras de empleo y bienestar social.

Con base en estos lineamientos, Salinas (2008), propone un modelo educativo para estar acordes con los requerimientos de la sociedad y los sectores productivos:

Figura 2. Propuesta de un modelo Educativo

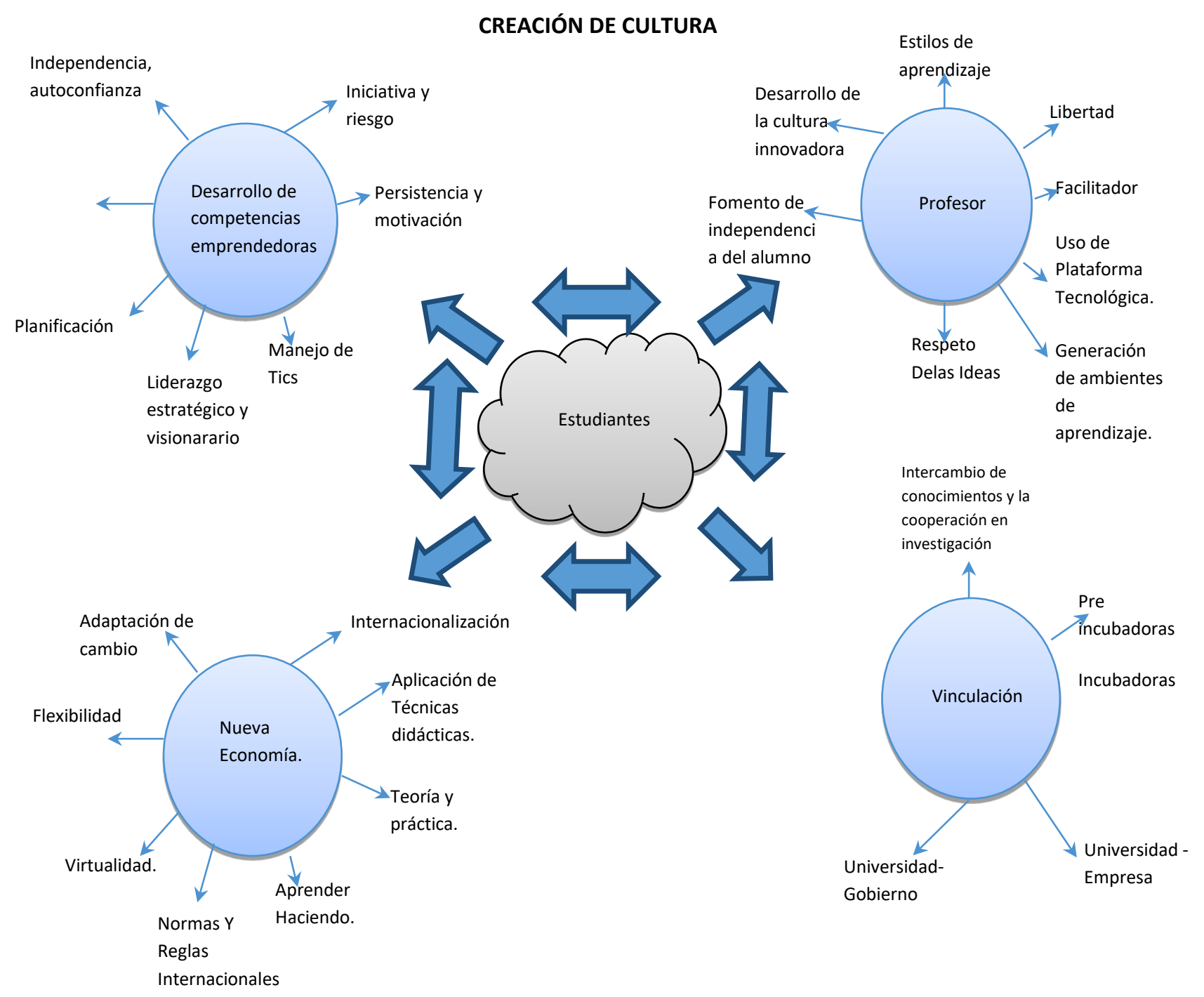

Fuente: Elaboración propia (2020). Basado en el razonamiento de Salinas (2008)

Como se observa, el modelo educativo se orienta en un sentido integrador, donde el auto aprendizaje del estudiante debería generar una cultura emprendedora con independencia y creatividad.

En torno a este aspecto aparecen cuatro entidades divididas en: Profesor; vinculación, nueva economía y desarrollo de habilidades. 
El autor de la investigación, considera estas vertientes importantes para tomar en cuenta en el modelo objeto del presente estudio, por cuanto presenta una visión integradora y parte de este análisis, sumado a nuestro aporte, se consideran para el desarrollo del modelo de gestión teórico académico.

La entidad profesor, hace referencia a que éste debe fungir como facilitador o guía orientador del proceso interactivo de enseñanza-aprendizaje, no deja de ser el experto en el tema, sin embargo, el alumno en los ambientes de aprendizaje pueda ser capaz de construir la generación de conocimientos, resolver problemas, utilizar capacidades de creatividad e innovación, tener respeto por sus ideas y libertad de opinión, un ambiente que no sea fastidioso para su desarrollo y que al contrario genere motivación al estudiante.

Es necesario establecer relaciones entre educación, investigación, negocios, industria, gobierno y comunidad para lograr subsistir en el ambiente global, siendo una de las necesidades actuales de la educación en el Ecuador.

\section{Resultados.}

La información recogida con respecto a las entrevistas semiestructuradas a las principales autoridades de la Universidad Católica de Cuenca, permitió determinar que en el ítem $\mathrm{N}^{\circ} 1$, que hace referencia a la dimensión del Modelo de gestión académica, en las Unidades Académicas de la Universidad Católica de Cuenca, las tres autoridades coinciden que existen políticas, y que la Universidad debe en impulsar el emprendimiento y la innovación.

Con respeto, al ítem $\mathrm{N}^{\circ} 2$, con relación a las competencias en la formación de los estudiantes referentes el aprendizaje del espíritu emprendedor, las autoridades consideraron que las competencias como la autoestima, la tecnología, iniciativa, liderazgo, fijación de metas, la creatividad, las destrezas y la decisión, competencias que deben caracterizar la formación emprendedora.

El ítem $\mathrm{N}^{\circ} 3$, con respecto a la dimensión política de emprendimiento innovador, en relación a los tipos de proyectos que ayudarían a la formación emprendedora, todas las autoridades coincidieron que es necesario impulsar proyectos de innovación y emprendimiento, que es la ruta a seguir para crear una actitud en las estudiantes, una cultura y una capacidad en la generación de ideas de negocios y empresas y una característica del entorno competitivo.

Asimismo, el ítem $\mathrm{N}^{\circ} 4$, sobre proyecto de emprendimiento en la UCACUE, las autoridades indicaron que actualmente no existe ningún proyecto, pero que la Universidad cuenta un laboratorio llamado FABLAB, que es un laboratorio que proporciona a sus usuarios el acceso a medios modernos para la creatividad, por varias máquinas controladas en ordenadores que permitan crear objetos físicos, maquetas, prototipos, entre otros. 
En cuanto al ítem $\mathrm{N}^{\circ} 5$, al respecto a promover la cooperación a través de la integración productiva, las autoridades entrevistadas manifestaron que el uso de las tecnologías de la información y comunicación, recursos humanos, económicos y financieros, redes de investigación, además de los convenios.

La dimensión de elementos curriculares, en el ítem $\mathrm{N}^{\circ} 6$, se preguntó a las autoridades sobre modelo triple hélice (Universidad-Empresa-Gobierno), las mismas indicaron que se está trabajando la integración de diferentes agentes, es una articulación productiva que favorece al incremento de la productividad en base a convenios y proyectos estratégicos.

El ítem $\mathrm{N}^{\circ} 7$, con respecto al emprendimiento como eje transversal en la malla curricular, sostuvieron que el emprendimiento tenga transversalidad en las mallas curriculares, de allí que se hace necesario su aplicación, para lo cual es necesario trabajar en cultura emprendedora, además de competencias y habilidades emprendedoras.

Igualmente, el ítem $\mathrm{N}^{\circ} 8$, con respecto al modelo de desarrollo y fortalecimiento de la cultura emprendedora, las autoridades manifestaron que están trabajando sobre el tema e identificando oportunidades para dotar de los recursos.

El ítem $\mathrm{N}^{\circ}$ 9, con respecto al modelo pedagógico, las autoridades indicaron que está en correspondencia con la cultura emprendedora, porque el emprendimiento permite desarrollar capacidades y ayuda a los estudiantes, a desarrollar su talento, para lograr sus objetivos, y para ella se está realizando la planificación respectiva.

Por su parte, el ítem $\mathrm{N}^{\circ} 10$, con respecto a la formación del personal docente que imparta las cátedras de emprendimiento e innovación, sostuvieron que es importante la capacitación y la actualización permanente, porque ello garantiza la calidad del docente para una mejor búsqueda de oportunidades e iniciativas.

Por último, el ítem $\mathrm{N}^{\circ} 11$, que hace referencia a trabajo grupal y la generación de normativa, facilitará el fortalecimiento en materia de emprendimiento e innovación, al respecto las autoridades entrevistadas revelaron que entre las políticas considero importantes las cuatro A, Asequibilidad, Accesibilidad, Adaptabilidad, Aceptabilidad definidas en el estatuto orgánico vigente, así como los principios expresados en el mismo documento, pero es necesario también el intercambio de experiencias y programas y fomentar el desarrollo e impulso del capital emprendedor, todo ello bajo una planificación adecuada.

\section{Contraste de datos o cruce de información.}

Un párrafo de comparación y contraste tiene por característica enfrentar dos ideas o conceptos. Se compara las similitudes y destacan las diferencias que puedan resultar.

La contrastación es una técnica utilizada de manera específica, para analizar los datos cualitativos y cuantitativos. En torno a este aspecto Pérez y Merino (2014), describen que el 
contraste es la acción y efecto de comprobar, es decir mostrar condiciones opuestas o diferencias marcadas, y/o probar la exactitud de algo. El contraste, por lo tanto, puede ser la oposición o contraposición que existe entre cosas o personas. De allí que es un camino hacia nuevas formas de producción del conocimiento y de concepción de la realidad.

En esencia, la contrastación representa el objetivo del investigador en la búsqueda de patrones de convergencia para poder desarrollar o corroborar una interpretación global del fenómeno humano objeto de la investigación y no significa que se tengan que utilizar métodos, fuentes de datos, investigadores, teorías o ambientes; comprende el uso de varias estrategias al estudiar un mismo fenómeno, por ejemplo, el uso de varios métodos, la técnica es la encuesta y el instrumento es el cuestionario y para el caso de las entrevistas, la técnica es la entrevista y el instrumento el guión de preguntas semi-estructuradas.

Los datos contenidos en la tabla de contrastación de información recogida permitió, determinar dentro de la dimensión Modelo de Gestión Académica, los ítems $\mathrm{N}^{\circ} 1$ y 2 , la información proporcionada por los entrevistados 1, 2 y 3 es similar en el primer caso existen políticas, pero faltan promover el emprendimiento y la innovación y en el segundo caso las autoridades consideran competencias como la autoestima, la tecnología, iniciativa, liderazgo, fijación de metas, la creatividad, las destrezas y la decisión, competencias que deben caracterizar la formación emprendedora.

Por su parte, con respecto a la dimensión política de emprendimiento innovador, se consideraron los ítems $\mathrm{N}^{\circ} 3,4,5$ y 6 , el primero las autoridades coinciden que es necesario impulsar proyectos de innovación y emprendimiento, la segunda que no existe ningún proyecto, la tercera que es importante el uso de las tecnologías de la información y comunicación, recursos humanos, económicos y financieros, redes de investigación, entre otros y el cuarto se manifiestas que se está trabajando la integración de diferentes agentes, en una articulación productiva que favorece al incremento de la productividad.

En cuanto, a la dimensión elementos curriculares, se encuentran los ítems $\mathrm{N}^{\circ}$ 7, 8, 9, 10 y 11 , el análisis indicó que el emprendimiento debe ser transversal en las mallas curriculares, se manifestó que se está trabajando sobre el tema e identificando oportunidades para dotar de los recursos, sobre el modelo pedagógico, las autoridades indicaron que está en correspondencia con la cultura emprendedora, porque el emprendimiento permite desarrollar capacidades y ayuda a los estudiantes; sobre la formación del personal docente que imparta las cátedras de emprendimiento e innovación, es importante la actualización continua, porque garantiza la propiedad del educador, para ello es importante determinar lineamientos, políticas y normas, y dotar de recursos para su aplicación.

Tabla 1. Contrastación de encuestas a autoridades de la UCACUE. 


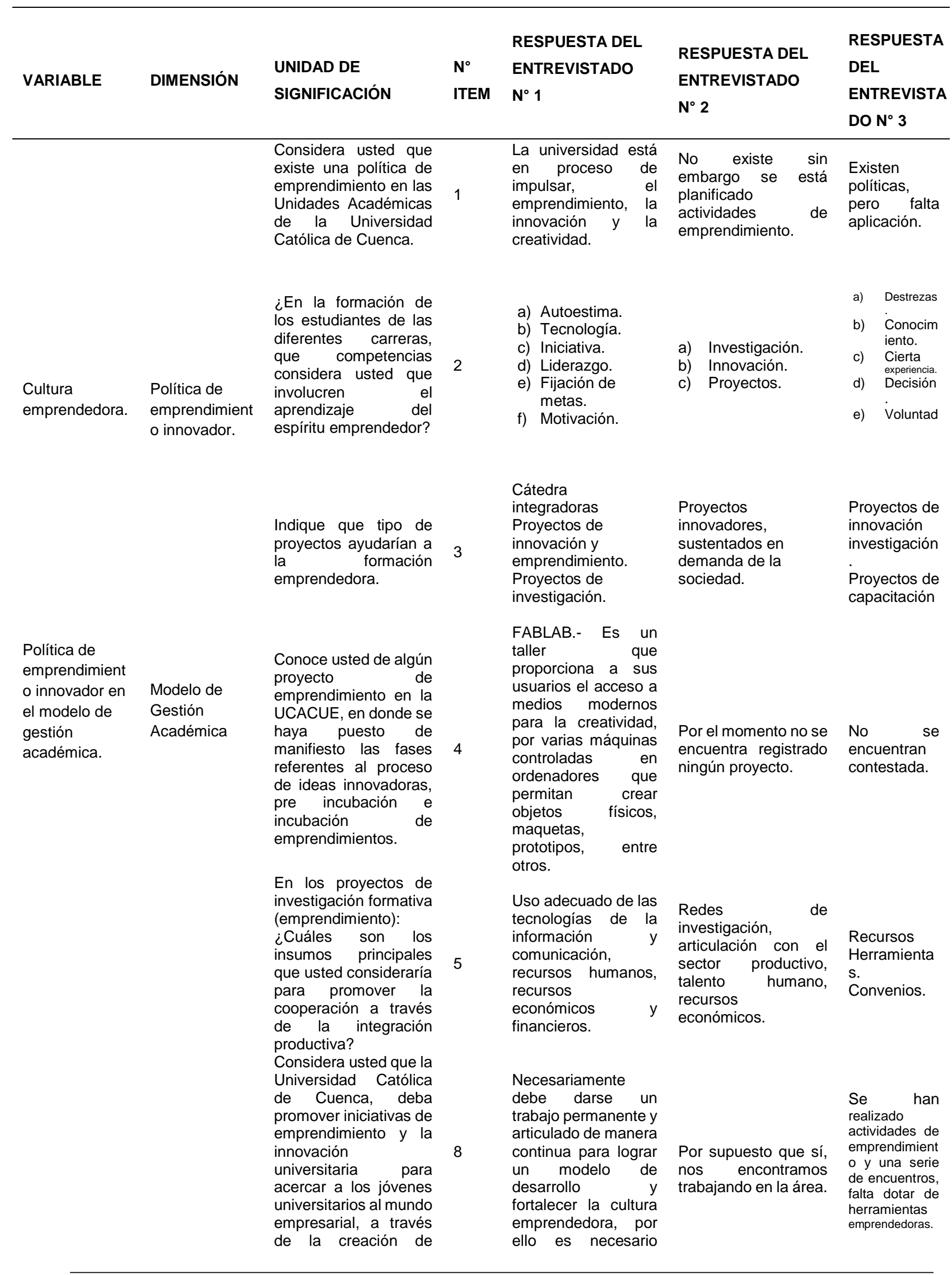




\begin{tabular}{|c|c|c|c|c|c|c|}
\hline VARIABLE & DIMENSIÓN & $\begin{array}{l}\text { UNIDAD DE } \\
\text { SIGNIFICACIÓN }\end{array}$ & $\begin{array}{l}\mathrm{N}^{\circ} \\
\text { ITEM }\end{array}$ & $\begin{array}{l}\text { RESPUESTA DEL } \\
\text { ENTREVISTADO } \\
\mathbf{N}^{\circ} 1\end{array}$ & $\begin{array}{l}\text { RESPUESTA DEL } \\
\text { ENTREVISTADO } \\
\mathbf{N}^{\circ} 2\end{array}$ & $\begin{array}{l}\text { RESPUESTA } \\
\text { DEL } \\
\text { ENTREVISTA } \\
\text { DO N } 3\end{array}$ \\
\hline & & $\begin{array}{l}\text { plataformas de apoyo } \\
\text { técnico a los } \\
\text { emprendimientos en } \\
\text { las etapas de gestión, } \\
\text { así como promover } \\
\text { encuentros a con } \\
\text { emprendedores. }\end{array}$ & & $\begin{array}{l}\text { identificar } \\
\text { oportunidades y } \\
\text { dotar de los recursos } \\
\text { para transformar en } \\
\text { ideas para hacer } \\
\text { algo nuevo. }\end{array}$ & & \\
\hline & & $\begin{array}{l}\text { Considera usted que el } \\
\text { modelo pedagógico } \\
\text { constructivista se } \\
\text { encuentra en } \\
\text { correspondencia con } \\
\text { la política de una } \\
\text { cultura emprendedora } \\
\text { en la Universidad } \\
\text { Católica de Cuenca. }\end{array}$ & 9 & $\begin{array}{l}\text { Si el modelo } \\
\text { pedagógico está en } \\
\text { correspondencia de } \\
\text { la cultura } \\
\text { emprendedora, } \\
\text { porque el } \\
\text { emprendimiento } \\
\text { permite desarrollar } \\
\text { capacidades y ayuda } \\
\text { a los estudiantes, a } \\
\text { desarrollar su } \\
\text { talento, para lograr } \\
\text { sus objetivos. }\end{array}$ & SI & $\begin{array}{l}\text { Los modelos } \\
\text { no son } \\
\text { sinónimos de } \\
\text { emprendimient } \\
\text { o, lo que } \\
\text { determina el } \\
\text { emprendimient } \\
\text { o es la } \\
\text { decisión, la } \\
\text { constancia y la } \\
\text { planificación. }\end{array}$ \\
\hline & & \begin{tabular}{lr}
\multicolumn{2}{l}{ Considera usted que el } \\
personal docente de \\
formación & y \\
capacitación & que \\
imparta las cátedras \\
especificadas para \\
emprendimiento deba \\
contar con un \\
certificado & de \\
formación & y \\
actualización & de \\
conocimientos en & en \\
metodologías de & de \\
emprendimiento e e \\
innovación, avalado \\
por un ente de \\
capacitación \\
acreditado por el \\
órgano competente.
\end{tabular} & 10 & $\begin{array}{l}\text { Necesariamente } \\
\text { debe ser así, es } \\
\text { importante que estén } \\
\text { debidamente } \\
\text { capacitados, para } \\
\text { una mejor búsqueda } \\
\text { de oportunidades e } \\
\text { iniciativa. }\end{array}$ & $\begin{array}{l}\text { Si, Pues garantiza la } \\
\text { formación y calidad } \\
\text { del docente. }\end{array}$ & $\begin{array}{l}\text { De ser } \\
\text { necesario si, } \\
\text { que conozca } \\
\text { del tema, y } \\
\text { tenga } \\
\text { experiencia. }\end{array}$ \\
\hline & & $\begin{array}{l}\text { ¿Según su criterio, que } \\
\text { políticas académicas } \\
\text { consideraría como } \\
\text { claves para que los } \\
\text { estudiantes tengan el } \\
\text { éxito como } \\
\text { emprendedores? }\end{array}$ & 11 & $\begin{array}{lr}\text { El trabajo grupal y la } \\
\text { generación } & \text { de } \\
\text { normativa, facilitara el } \\
\text { fortalecimiento en } \\
\text { materia } & \text { de } \\
\text { emprendimiento e } \\
\text { innovación, además el } \\
\text { intercambio r de } \\
\text { experiencias r y } \\
\text { programas. Fomentar } \\
\text { el desarrollo e impulso } \\
\text { del capital } \\
\text { emprendedor. }\end{array}$ & $\begin{array}{l}\text { Entre las políticas } \\
\text { considero } \\
\text { importantes las } 4 \mathrm{~A} \text {, } \\
\text { Asequibilidad, } \\
\text { Accesibilidad, } \\
\text { Adaptabilidad, } \\
\text { Aceptabilidad } \\
\text { definidas en el } \\
\text { estatuto orgánico } \\
\text { vigente, así como los } \\
\text { principios } \\
\text { expresados en el } \\
\text { mismo documento. }\end{array}$ & $\begin{array}{l}\text { Determinar } \\
\text { lineamiento, } \\
\text { políticas, } \\
\text { normas, } \\
\text { dotar de } \\
\text { recursos y } \\
\text { talento } \\
\text { humano todo } \\
\text { bajo una } \\
\text { planificación. }\end{array}$ \\
\hline
\end{tabular}

Fuente: Autoría propia (2020)

En el presente trabajo de investigación el proceso de contrastación permitió la construcción del Modelo de Gestión Teórico- Académico para el Desarrollo de Políticas y Programas de 
Emprendimiento Innovador en la Universidad Católica de Cuenca, todo ello, en correspondencia con los objetivos de investigación.

\section{Conclusión.}

- De la información recogida a las autoridades entrevistadas, con respecto a las normas legales, planes de desarrollo y reglamentación de la UCACUE, las mismas se están trabajando para adecuar al nuevo Estatuto Orgánico aprobado por el Consejo de Educación Superior (CES) mediante Resolución N RPC- SO-24-No.406-2019 de fecha 10 de julio de 2019.

- Al examinar el estado del arte de la cultura emprendedora innovadora en el Ecuador y su vinculación con el Plan Nacional de Desarrollo Toda una Vida, las Agenda Zonal 6, y los Planes de Desarrollo y Ordenamiento Territorial, las autoridades entrevistadas sostuvieron que la empresa está vinculada a la formación y al empleo, por tanto las Universidades están respondiendo a la demanda de formación en emprendimiento de sus estudiantes, indicaron que se requiere un trabajo más articulado con las bolsas de empleo existentes en Cuenca (Cámara de la Producción, Universidades, Ministerio de Relaciones Laborales, entre otros), las cuales vinculan las demandas de las empresas con los recién graduados, que es la gente preparada, colaboradora y propositiva.

- Con respecto a la vinculación y modelo triple hélice, los actores coinciden, que la relación Universidad- empresa, es la de mayor importancia, por cuanto permitirá potenciar las capacidades de la población estudiantil, mediante pasantías, practicas pre profesionales, talleres, eventos, y asesoría personalizadas.

- Respeto a las opiniones emitidas por los actores universitarios, es factible construir, un modelo de gestión teórico- académico para el desarrollo de políticas y programas de emprendimiento innovador en la Universidad Católica de Cuenca, para ello fue imperioso la aplicación del emprendimiento como uno de los ejes transversales del proceso Universitario, basado en la creación de valor agregado en las metodologías académicos curriculares y extracurriculares de la política Universitaria, integrada de manera estructural y vinculada al desarrollo sostenible de la Región 6.

- Con respecto al modelo pedagógico de la UCACUE, de acuerdo a las entrevistas realizadas a las principales autoridades de la Universidad Católica de Cuenca, sostienen que está en correspondencia con la cultura emprendedora, porque el emprendimiento permite desarrollar capacidades y ayuda a los estudiantes, a desenvolver su talento, para lograr sus objetivos, y para ella se está realizando la planificación respectiva y la adaptación de políticas al nuevo estatuto orgánico institucional, considerando, la asequibilidad, accesibilidad, adaptabilidad, aceptabilidad, en articulación con los ejes sustantivos docencia, investigación y vinculación con la sociedad.

- La Universidad Católica de Cuenca hasta el mes de diciembre del año 2018, impulso actividades de emprendimiento en las Unidades Académicas tales como proyecto 
plaza, proyecto del adulto mayor, capacitación a estudiantes y docentes en temas de cultura emprendedora, desde esta fecha hasta la presente no ha existido actividades con "real enfoque en Emprendimiento", si sus objetivos tenían relación con el proceso de emprender, crear una empresa, el uso de herramientas como plan de negocio, propuesta de valor y modelo de negocio, habilidades, características y competencias emprendedoras, hoy se encuentra en un nivel neutro hasta la toma de decisiones de las autoridades de la Universidad.

- En cuanto al desprendimiento de empresas (spin-offs), en un futuro cercano, la propuesta y estrategia es establecer un programa de incentivos para el desprendimiento de empresas que pudieran generarse en la UCACUE y hacer campañas de difusión en el interior de las Unidades Académicas, dando información al respecto. Un programa de incentivos no implica que la UCACUE sea accionista de la empresa, ni participar directamente en la constitución de su capital, el objetivo es desprender, no poseer empresas.

- La validación del modelo, se realizó mediante juicio de expertos, integrado por un representante de la academia, un representante del sector gubernamental y un representante de los sectores productivos, según los criterio de factibilidad curricular, precisión, utilidad, ética, pertinencia, y contenido, existe correspondencia de los elementos de gestión de emprendimiento con los enfoques teóricos y el marco constitucional y legal, de igual manera, relaciona los elementos y procesos de gestión con el modelo teórico - académico, así mismo se articulan las competencias emprendedoras con los contenidos del modelo de gestión, y por último establecen las políticas de emprendimiento y relación con la realidad regional, local e institucional, por lo tanto permite determinar la pertinencia e implementación del modelo.

- La Universidad Católica de Cuenca, una vez que ha definido su estatuto orgánico, debe precisar la política institucional Universitaria, integrada de manera estructural y vinculada al desarrollo sostenible y sustentable del territorio.

- La UCACUE y sus respectivas Unidades Académicas deben generar conciencia integradora, así como apoyar el proceso académico para formar profesionales competentes, con pensamiento emprendedor e innovador, con pasión y compromiso, para crear y liderar sus propias empresas de base social, economía general y base tecnológica; sobre los principios de un modelo educativo, que promueve la investigación, creatividad, innovación, productividad y competitividad.

- El contenido de los cursos pedagógicos debe enfocarse en liderazgo, las tecnologías de la información y comunicación y los negocios, resaltando el papel de la creatividad e innovación en un ambiente competitivo, y utilizando una pedagogía que involucre trabajo en equipo y que, sobretodo, sea motivante.

\section{Referencias bibliográficas.}

Arias, F. (2016). El Proyecto de Investigación: Introducción a la metodología

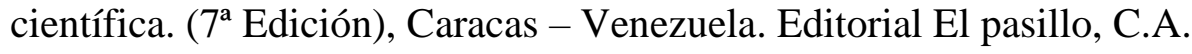


Arroyo, M. (2016). Emprendimiento y Universidad Emprendedora: conceptualización, propuesta metodológica y caracterización de la Universidad Politécnica de Valencia, Universidad Politécnico de Valencia, Recuperado de: file:///C:/Users/ZONA\%20INFORMATICA/Desktop/CAPITU LO\%20II\%2020-042018/universidad\%20emprendedora\%20oK.pdf

Carland, J. W., Hoy, F., y Carland, J. A. C. (1988). Who is an entrepreneur? is a question worth asking. American Journal of Small Business, 12(4), 33-39.

Drucker, P. (1985). La Innovación y el empresario innovador. Ed. Edhasa Página. 25-26; $35-44$.

Harbison, F. (1956). Entrepreneurial organization as a factor in economic development. The Quarterly Journal of Economics, 364-379.

Hernández, S.; Fernández, C.; Baptista, L. (2016). Metodología de la Investigación. México: Mc Graw Hill Educación, 6ta. Edición.

Hurtado de Barrera, J. (2010). Guía para la comprensión holística de la ciencia, Tercera Edición, Fundación Sypal: Caracas-Venezuela.

Naciones Unidas, Asamblea General (2012). "Iniciativa empresarial para el desarrollo" realizada el 21 de diciembre de 2012, pág. 67/202.

La Agenda 2030 y los Objetivos de Desarrollo Sostenible, (2016). Una oportunidad para América Latina y el Caribe, Publicación de las Naciones Unidas, mayo de 2016, Impreso en Santiago.

Pérez, L. (2016). Propuesta metodológica para la caracterización de pautas emprendedoras en materia de emprendimiento universitario. Validación en el Campus d'Alcoi de la Universitat Politécnica de Valencia, Recuperado de: file:///C:/Users/ZONA\%20INFORMATICA/Desktop/CAPITULO\%20II\% 2020-042018/TESIS\%20DOCTORALES- MARCO\%20TEORICO/Pérez\% 20\%20Propuesta\%20metodológica\%20para\%201a\%20caracterización\%2 0de $\% 20$ pautas $\% 20$ emprendedoras $\% 20$ en $\% 20$ materia $\% 20$ de $\% 20$ empr $\% 20 \mathrm{ok}$.pdf

Pérez, J. y Merino, M. (2014). Definición de contraste, recuperado de: (https:// definicion.de/contraste/)

Rasheed, H. 2000. Developing Entrepreneutial Potencial in Youth of Entrepreneurial Education and Venture Creation. Disponible en 
http://www.usasbe.org/knowledge/proceedings/2001/063.pdf.

Salinas, P.(2008). Desarrollo de Habilidades de Innovación y Creatividad en Estudiantes

Universitarios de Ingenierías, de la Universidad Tecnológica de Monterrey México, Recuperado de: file://C:/Users/ZONA\%20INFORMATICA/Desktop/CAPITULO\%20II\%2020-042018/DocsTec_Innovaciòn\%20educativa.pdf.

Santa Palella y Feliberto Martins (2012). Metodología de la Investigación Cuantitativa, 3ra edición, FEDUPEL. Caracas-Venezuela.

Schumpeter, J. A. (1942). Socialism, capitalism and democracy. Harper and Brothers.

Shane y Venkataraman, (2017). ¿Fomentar el espíritu empresarial universitario? El efecto del Bayh-Dole Acton.

Tejada. J. (1997). El proceso de Investigación científica. Barcelona-España, Editorial FUND. Caixa de Pensions.

UNESCO. (2015). Foro Mundial sobre Educación 2015. Educación 2030: Hacia una educación inclusiva y equitativa de calidad y un aprendizaje a lo largo de la vida para todos. Corea: UNESCO.

Villar, F. (2015). Los estilos de aprendizaje y su aplicación en la formación para El emprendimiento, de la Universidad de Burgos, Facultad de Educación, Burgos-España, Recuperado de: file://C: Desktop/CAPITULO\%20II\%2020-042018/TESIS\%20DOCTORALES-

MARCO\%20TEORICO/Villar_Mata\%20cREATIVIDAD\%20Y\%20EMPRENDIM IENTO\%20OK.pdf.

Wennekers, S., y Thurik, R. (1999). Linking entrepreneurship and economic growth.

Small Business Economics, 13(1), 27-56.

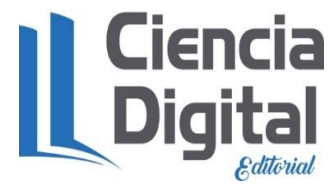


PARA CITAR EL ARTÍCULO INDEXADO.

Becerra Molina, E. (2020). Fomento de iniciativas de emprendimiento y la innovación para acercar a los jóvenes universitarios al mundo empresarial. Ciencia Digital, 4(4), 65-84. https://doi.org/10.33262/cienciadigital.v4i4.1430

\section{\Ciencia}

El artículo que se publica es de exclusiva responsabilidad de los autores y no necesariamente reflejan el pensamiento de la Revista Ciencia Digital.

El artículo queda en propiedad de la revista y, por tanto, su publicación parcial y/o total en otro medio tiene que ser autorizado por el director de la Revista Ciencia Digital.
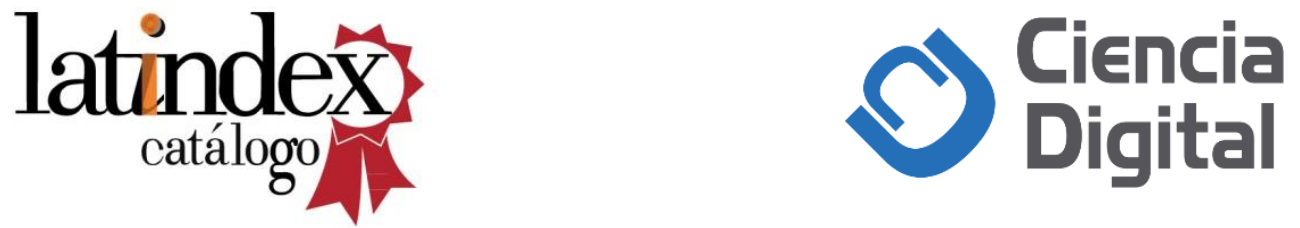